\title{
Bringing a New Light on Old Problems - Interview with Laure Saint-Raymond
}

Roberto Natalini (Consiglio Nazionale delle Ricerche, Rome, Italy, Chair of the Raising Public Awareness Committee of the EMS)

Laure Saint-Raymond is a French mathematician working in partial differential equations, fluid mechanics and statistical mechanics. She is a professor at École Normale Superieure de Lyon. In 2008, she was awarded the EMS Prize and, in 2013, when she was 38 years old, she became the youngest member of the French Academy of Sciences.

\section{Roberto: Let me start with a very trivial question: when did you become interested in mathematics? \\ Laure: Actually, it was quite late. In high school, I was a good student but somehow I was more interested in mu- sic. But, being good in maths, as was the norm in France, I entered the so-called "Classes préparatoires" (which is preparation for entrance selection for the "Grandes Écoles") and then the École Normale Supérieure (ENS) in Paris. Here, I found very enthusiastic teachers and so my interest for mathematics started.}

How were your parents involved in your interest in mathematics? Did you have an important teacher before university?

I had a maths teacher during the "Classes préparatoires" with a strong passion for mathematics and, in particular, for logic. However, even though my father is a mathematician, I was not really pushed by my parents to go in this direction. I was quite free to make my choice.

In the ENS, I found many inspirational professors, like the physicist Yves Pomeau, who used to introduce baby models to catch important physical phenomena such as the growth of trees. On the mathematical side, I should mention Yann Brenier, with his very original way of seeing all things, and Henry Berestycki. And, finally, it was with François Golse that I really discovered the connection between mathematics and physics or, to say it better, how to couple the rigour of maths with the inspiration arising from physics.

What are your main fields of interest in mathematics and how and why did you start to work on them?

I started my research in plasma theory, looking at the qualitative behaviour of beams of charged particles in strong magnetic fields. The approach was driven by kinetic theory methods, with a deep interplay of mathematics and physics. In collaboration with my PhD advisor François Golse, we solved one part of Hilbert's sixth problem. This problem consists of developing mathematically "the limiting processes [merely indicated in Boltzmann's

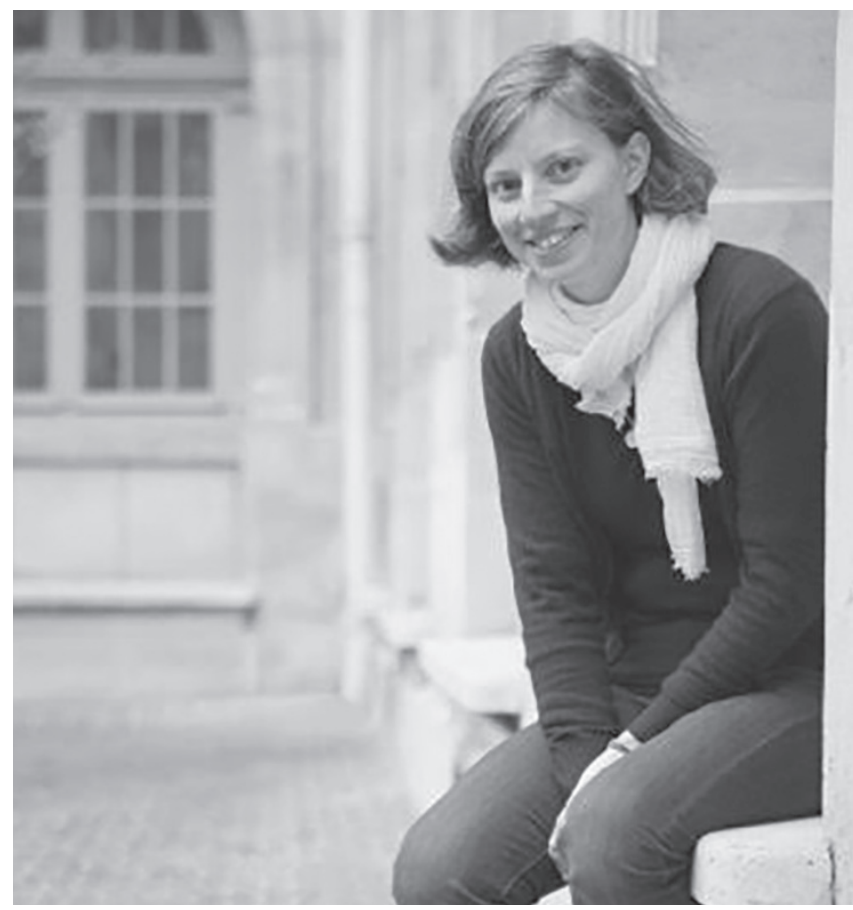

Laure Saint-Raymond at ENS Paris, 2014. (Pboto: CNRS)

work] which lead from the atomistic view to the laws of motion of continua".

What we established is the rigorous transition from the Boltzmann kinetic description, where the gas is considered as a collection of interacting particles described statistically, to a fluid description given by the Navier-Stokes equations, where the flow is described only through macroscopic quantities such as the average speed or the pressure of the fluid.

What have been your main original ideas in proving the limit from Boltzmann to the Navier-Stokes equations? Actually, I have contributed in both collecting and organising in an original way many existing techniques, and in developing some new mathematical tools, such as the so-called $L^{1}$ velocity averaging lemma related to dispersion and mixing. The Boltzmann equation describes the state of a gas using a distribution function that depends on space, velocity and time. It expresses a balance between two mechanisms: the transport and the collisions. This equation has no regularising effect and so, if we have a singularity in the solution, we keep it forever. And 
this is a problem when you study the fast relaxation limit (i.e. the asymptotic behaviour when the relaxation to local equilibrium due to collisions is much faster than the transport that correlates close positions) because you need some compactness.

It was noticed by Golse, Lions, Perthame and Sentis that observables, which are obtained by taking averages with respect to the velocity variable, are more regular than the solution itself. We were able to combine this result with hypoelliptic properties of the transport to prove that if you gain some nice behaviour in the velocity then you can gain something also in the space variable. This was one of the main tools to prove our convergence result.

\section{What about some other problems you have considered?}

The other part of my work is concerned with large-scale geophysical flows where the Coriolis force is dominant, taking into account the dominating influence of the Earth's rotation. Classical methods for linear singular perturbation problems fail when the oscillations cannot be described explicitly because one does not even know whether the waves will be captured or dispersed. For instance, close to the equator, the spatial variations of the Coriolis acceleration cannot be neglected. The spectral structure of the propagator is completely modified and one can prove that fast oscillations are trapped in a thin band of latitudes.

Another challenging problem is to understand the interaction with the boundaries, which is responsible for most energy exchanges (forcing and dissipation), even though it is concentrated in very thin layers close to the bottom and the surface.

Now, I try to understand the propagation of internal and inertial waves in the ocean, in regions with a variable topography. I collaborate with physicists to understand how to separate the different time and space scales, neglecting the very complex dynamics at small scales but keeping the qualitative behaviour of the solutions.

\section{Are you still working on Hilbert's sixth problem?}

Yes, of course! More recently, mainly in collaboration with Isabelle Gallagher and Thierry Bodineau, I have worked on the full problem, namely, to make a rigorous derivation of fluid models from particle models, which I think is a much more difficult problem. A very challenging question is to explain the appearance of irreversibility at the macroscopic level. At this stage, there is no general theory but some special results have been obtained. For instance, we were able, under some specific scaling assumptions, to obtain the Stokes equations directly as the limit of particle models. It is not the optimal result but it is the first rigorous derivation of fluid equations from Newton's mechanics. Our starting point is solely the deterministic collisions of hard spheres, coupled with a suitable entropy bound. However, it is quite clear that we cannot hope to obtain the full result, i.e. the convergence to the Navies-Stokes equations, using the same ideas. So, we are looking around for some new ideas.

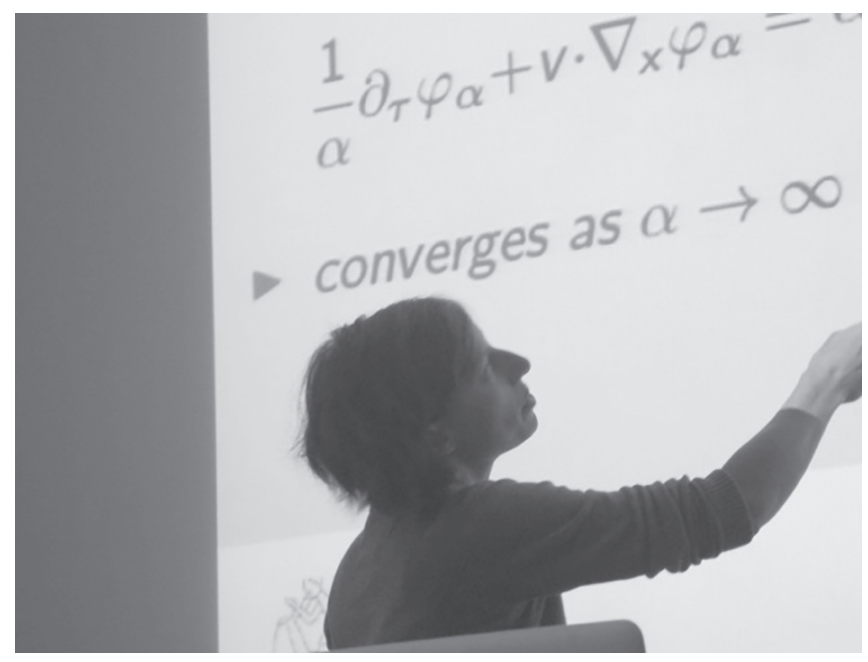

Laure at the 23rd International Conference on Discrete Simulation of Fluid Dynamics, July 29, 2014, École Normale Supérieure, Paris.

You have been awarded with many prizes. Which one is the most important for you?

First, I have to say that when you receive a prize, you then receive a lot of them, which does not mean that you have more merit. Of course prizes come as recognition from the mathematical community and I am very proud of the EMS Prize I received in 2008. But I think that prizes should be overall understood as an encouragement to go further and maybe to take more risks and more responsibilities.

Speaking of responsibility, I remember your intervention in 2015 about publications, during the event for the 25th anniversary of the EMS at the Institut Poincaré in Paris.

Yes, I am really concerned by this point. I believe that, as a mathematical community, we really publish too much and that senior people with accomplished careers should be more careful and selective when submitting papers. Most of the time, nobody reads these papers and it is even difficult to find somebody to do a good peer review. Myself, I have adopted as a rule to referee at least twice the number of papers that I publish each year. It is crucial to properly review the papers and also to read and discuss articles from other researchers. This is the only way to be a scientific community.

I believe that science is a common project and not an isolated enterprise. On the other side, unfortunately, we are faced by all these national and international rankings, which are very often quite meaningless and based on quantitative metrics. Nobody is interested in what people are really doing and I think it is bad for mathematics.

\section{How much in your work is intuition and how much is just hard work? \\ The starting point of each of my papers is about trying to bring a new light on a problem. Unfortunately, many of my papers are a mess of technical details but still we try to explain one or two new ideas. In this sense, my works}


are not only technical but there is always some intuition to be made rigorous. You have an idea and then you try to work out the details and you struggle with some problems. And to solve these problems, you have to understand something that you missed before. You don't fully understand it until you have a complete proof. This is, in my opinion, the essence of mathematical work.

How do you organise your work? Do you follow a routine or does it vary a lot according to external conditions?

I work most of the time with the same collaborators, since it takes a lot of time to share the same language, the same feelings on the topics and so on. I'm not the kind of person who goes to a conference, meets some people and immediately starts a new collaboration.

Two years ago, I spent a sabbatical in the US, where I had a lot of time and no duties. It was really quiet and I had a great time working with no constraints but somehow it was not long enough to develop new collaborations.

How has it been important for you to be in Paris for many years?

For a very long time, we didn't leave Paris so as to stay close to our parents, who helped a lot with the children, and, I have to say, I didn't quite realise the great opportunity I had. Actually, in Paris, it is possible to discuss and collaborate with a lot of people with different backgrounds and ideas.

Out of Paris, you are maybe not exposed to such a large mathematical community but somehow it gives you more opportunities to meet people doing something really different and to go into new research directions. I have now moved to Lyon where I am very happy.

In France, women in mathematics are not so common, even if some things have changed in recent years. Could you explain the difficulties that women can sometimes experience in having a satisfactory career in mathematics?

Actually, I have to say that, in my experience, I didn't feel any discrimination against women. My impression is that somehow the problem is more in our society. One reason why women are not following scientific careers is maybe the French system of education based on selection and

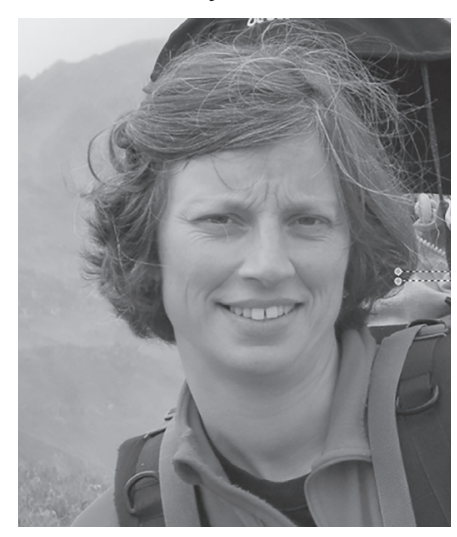

On a family holiday in the Alps. lems? You have a large family with six children. How is it possible to work so hard with a lot of children and commitments?

My husband is just great and makes everything at home [she smiles]. Also, for many years, our parents helped us taking care of the kids very often. Besides, the French school system (starting at the age of 3 ) is helpful in this regard.

But, nevertheless, for a long time, I needed to be at home at $5 \mathrm{pm}$ almost every day ... and I wrote fewer papers than most of my colleagues!

What do you do outside maths? Do you have hobbies? What do you like to do?

I do plenty of things like hiking and skiing and this is also one of the reasons why I like very much being in Lyon. Also, I enjoy music, playing the cello. Sometimes I even play chamber music with colleagues.

\section{A last question: what is your bedtime reading?}

It is hard to say; sometimes I just sleep [laughing]. But, for instance, I like very much Eric-Emmanuel Schmitt for his positive attitude about life. More generally, I look for books where I find a supplement of energy to live, something that helps to find the positive side of our lives.

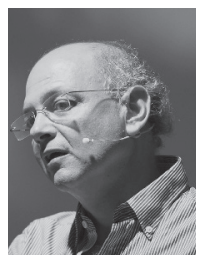

Since 2014, Roberto Natalini has been Director of the Istituto per le Applicazioni del Calcolo "Mauro Picone" of the National Research Council of Italy. His research interests include fluid dynamics, road traffic, semiconductors, chemical damage of monuments and biomathematics. He is Chair of the Raising Awareness Committee of the European Mathematical Society. 